\title{
Ultrafast Laser Formation of Nanoparticles Using Interfaces
}

\author{
Ryan D. Murphy', Michael J. Abere ${ }^{2}$, Ben Torralva ${ }^{2}$, Steven M. Yalisove ${ }^{2}$ \\ ${ }^{1}$ Applied Physics Program, University of Michigan, Ann Arbor, MI 48109 \\ ${ }^{2}$ Department of Materials Science and Engineering, University of Michigan, Ann Arbor, MI, 48109
}

Well-dispersed nanoparticles have been made by irradiating thin films of metals on transparent substrates in air with high intensity femtosecond laser pulses. The glassmetal interface is irradiated from the transparent side and the nanoparticles are collected on a substrate several hundred microns away from the structure. This geometry allows for direct printing of nanoparticles to devices without the need for water or deflocculants.

Interfaces have been found to play an important role in material removal after irradiation by femtosecond pulses. Only $10 \mathrm{~nm}$ of nickel sputter coated onto a silicon substrate changes the morphology of craters in the nickel after irradiation by $150 \mathrm{fs}$ pulses at a wavelength of $780 \mathrm{~nm}$ causing material removal to occur at fluences below the bulk removal threshold. Similar behavior is seen at metal-glass interfaces and initiation of material removal at the interface allows for nanoparticle formation at fluences an order of magnitude lower than those required when using nanosecond pulses. It will be shown that the thickness of the film controls the size distribution of nanoparticles, and characterization of the resulting nanoparticle morphology using Transmission Electron Microscopy will be presented. Device fabrication, such as $\mathrm{p}-\mathrm{n}$ junctions and ferroelectric layers, for solar applications will also be discussed. 